\title{
Elaboration and Magnetic Properties of Cobalt-Palladium Magnetic Nanowires Encapsulated in Carbon Nanotubes
}

\author{
Olivier Rousseau, Cynthia Locard, Aïchata Kane, Yves Roussigné, Samir Farhat*, \\ Salim Mourad Chérif*
}

Laboratoire des Sciences des Procédés et des Matériaux, CNRS, LSPM-UPR 3407, Université Paris 13, PRES Sorbonne-Paris-Cité, Villetaneuse, France

Email: *cherif@univ-paris13.fr, *samir.farhat@lspm.cnrs.fr

How to cite this paper: Rousseau, O., Locard, C., Kane, A., Roussigné, Y., Farhat, S. and Chérif, S.M. (2017) Elaboration and Magnetic Properties of Cobalt-Palladium Magnetic Nanowires Encapsulated in Carbon Nanotubes. Journal of Surface Engineered Materials and Advanced Technology, 7, 1-12.

http://dx.doi.org/10.4236/jsemat.2017.71001

Received: November 2, 2016

Accepted: December 19, 2016

Published: December 22, 2016

Copyright $\odot 2017$ by authors and Scientific Research Publishing Inc. This work is licensed under the Creative Commons Attribution International License (CC BY 4.0).

http://creativecommons.org/licenses/by/4.0/

\begin{abstract}
Bimetallic one-dimensional (1-D) cobalt-palladium magnetic nanowires encapsuled by carbon nanotubes were synthesized on silicon substrate using plasma enhanced chemical vapor deposition technique. After the deposition of the catalyst, the growth of nanotubes takes place in two stages. The first is a thermal pretreatment to transform continuous nanometer bimetallic thick film into isolated and uniformly distributed nanoparticles over the entire surface of the substrate. The second step results in the growth of nanotubes perpendicular to the substrate by the addition of carbon atoms on the insulated metal nanoparticles. While growing the nanotubes at given thermochemical conditions, a Co-Pd eutectic is thought to diffuse inside the cavity of the nanotube along a length of few hundreds of nanometers as determined by high resolution, spatially resolved Electron Energy Loss Spectroscopy (EELS), and energy filtered elemental mapping. The magnetic anisotropy along the nanotube directions is observed. Ferromagnetic or superparamagnetic-like behavior of the filled nanotubes was measured through local magneto-optical Kerr effect or global superconducting quantum interference device measurements, respectively. Information on the magnetism of filled nanotubes at different scales is pointed out and discussed.
\end{abstract}

\section{Keywords}

Magnetic Nanowire, Filled Carbon Nanotubes, Cobalt, PECVD, Static Magnetic Measurements

\section{Introduction}

Filling carbon nanotubes with chosen materials opens new possibilities of gene- 
rating nearly one-dimensional (1-D) objects with distinct properties and potential applications depending on both the chemical nature and spatial arrangement of the encapsulated guests. Due to their low dimensionality and large geometric aspect ratio, obtained nanowires exhibit different properties compared with their bulk counterpart. In the case of magnetic materials, the magnetic moment of the resulting anisotropic objects will tend to align with an "easy axis" which is an energetically favorable direction of spontaneous magnetization. Since the magnetic moment increases with decreasing nanowire thickness, we need to reduce the diameter of the nanowire. Open carbon nanotubes have been suggested as ultra-confined templates to encapsulate melted metals or salts via capillarity forces [1]. Indeed, their inherent geometrical properties such as their small diameter and long length associated with their electronic and nuclear spin degrees of freedom lead to quantum confinement effects that make them almost ideal one-dimensional (1D) systems with interesting consequences for electron and spin transport. However, due to the small cavity diameter of 1 to $10 \mathrm{~nm}$, the filling efficiency is very low, decreasing the length of the nanowires. Different filling methodologies were proposed using either single or multi walled carbon nanotubes CNT's as templates. These methods may be classified in two groups: (i) ex situ techniques necessitating an opening of the CNT by oxidation in air prior to their wetting by immersion in molten salts, or low-melting-point metal such as $\mathrm{Pb}$ or $\mathrm{Bi}$ [1] [2]. Since most of the metals have high-melting-point and high surface tension as high as $\gamma=1881 \mathrm{mN} / \mathrm{m}$ for cobalt and $\gamma=1482 \mathrm{mN} / \mathrm{m}$ for palladium; ex situ filling remains very difficult. (ii) An alternative approach is to fill the nanotube by capillarity forces in situ during their growth [3]. The recent developments of nanofabrication techniques allow access to individual CNTs opening the way toward spintronics [4] and electronics devices. Indeed, the first organic spintronic device was reported by Tsukagoshi et al. [5] in 1999 and was based on a multi-walled carbon nanotube (MWCNT) contacted by cobalt contacts in a conventional two-terminal spin valve geometry. Those interests lead to extensive research on how to control the CNT synthesis and their properties for the different applications. Several synthesis techniques were used including electrical arc discharge [6] [7], laser ablation [8] and chemical vapor deposition (CVD) [9] [10]. These elaboration methods are accompanied by characterization techniques that enable the mapping of the CNT characteristics for large sample sizes and populations. Some applications of CNTs such as field emission displays or photovoltaic cells, require a scalable production method. Hence, the most promising techniques, because of scalability and controllability, are CVD techniques. Plasmaenhanced chemical vapor deposition (PECVD), a variant of CVD, is an alternative approach for CNT synthesis. PECVD processing generally uses cold-wall design that prevents thermal damage to the substrate. Another advantage is the capability of obtaining relatively high deposition rates and more pure and uniform compounds by taking advantage of the high-energy electrons reactivity. CVD synthesis can be performed with catalyst [11] or catalyst free [12]. When transition metals such as $\mathrm{Co}, \mathrm{Ni}, \mathrm{Pd}, \mathrm{Pt}$ are used as catalyst, CVD-method can lead to 
self-assembled CNTs into aligned structures [13] [14] [15]. The distinctive organization of aligned CNTs is further governed by Van der Waals interaction between the tubes [16]. In 2004, a variant of CVD process called the super-growth CVD or water-assisted CVD was proposed by Hata group in Japan [17]. Water addition into the reactor enhances activity and lifetime of the catalyst avoiding the competing amorphous carbon formation pathway. With this method dense and well-aligned CNT forests, with heights up to $2.5 \mathrm{~mm}$ are produced [17].

It is very promising to increase the properties of CNTs depending on the aimed potential application. The most efficient way to control those additional properties of CNTs is to functionalize them. The functionalization is achieved either by chemical grafting on the outer surface or by filling the CNTs with materials which enhance the properties of interest. Several works report the filling of CNTs by magnetic nanoparticles post CNT synthesis or during the synthesis [18]. Opened CNTs have been suggested as ultra-confined templates to encapsulate melted metals or salts by using capillarity forces [19]. An alternative approach is to encapsulate one-dimensional foreign materials into CNTs during their growth.

In this paper, we used in situ filling method consisting in plasma enhanced chemical vapor deposition process, PECVD to successfully growth cobalt-palladium filled CNT forest. During the growth, the catalyst diffuses inside the CNTs filling them uniformly on a length of roughly $300 \mathrm{~nm}$ letting free the rest of the CNTs for hybrid organic-spintronic devices. The magnetic behavior of these CNTs was characterized by local magneto-optical Kerr effect (MOKE) as well as by global superconducting quantum interference device (SQUID) measurements. Because of CNT geometrical characteristics, shape anisotropy effects are pointed out.

\section{Experimental}

Carbon nanotube growth and filling typically involves three steps depicted in Figure 1. In the first step (i), a nanometer thick film catalyst of cobalt or bilayer cobalt-palladium is deposited under ultrahigh vacuum by e-beam evaporation on a $\mathrm{SiO}_{2} / \mathrm{Si}$ wafer. After its deposition, the catalyst was first thermally annealed in a vacuum chamber at a pressure of $2 \times 10^{-6} \mathrm{mbar}$ and a temperature of the order of $973 \mathrm{~K}$. As schematically shown in Figure 1, this step aims to disintegrate the continuous film onto isolated and self-organized metal nanoparticles. Then in order to chemically reduce the catalyst, the sample is exposed to hydrogen plasma at a pressure of $10 \mathrm{mbar}$ and a microwave power of 340 watts. The flow rate of $\mathrm{H}_{2}$ was maintained at $10 \mathrm{sccm}$ (standard cubic centimeter per minute) and $10 \mathrm{sccm}$ of methane $\mathrm{CH}_{4}$ mixed with $2 \mathrm{sccm}$ of oxygen $\mathrm{O}_{2}$ [17] are added in the plasma (under a regulated pressure of 10 mbar). Methane decomposition occurs through plasma activation with energetic electrons and gas temperature of $\sim 2200 \mathrm{~K}$ while the substrate temperature is maintained at $973 \mathrm{~K}$. The hydrocarbons obtained from the plasma decomposition of $\mathrm{CH}_{4}$ lead to the growth of the CNTs perpendicularly to the substrate by the addition of carbon atoms 


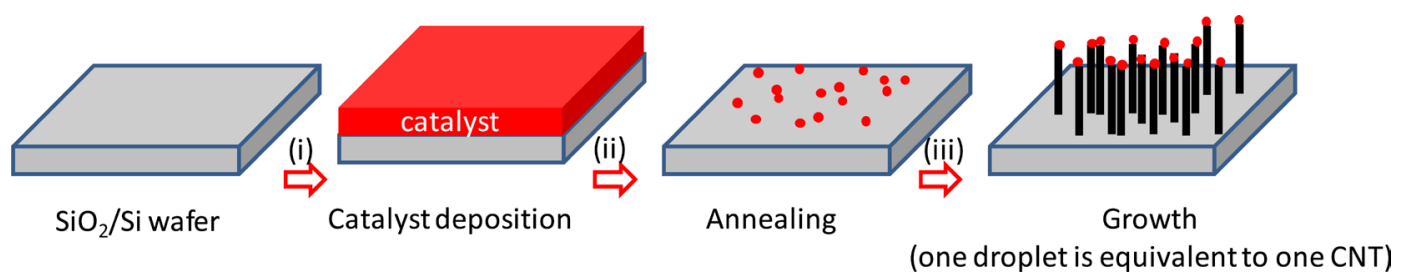

Figure 1. Schematic stages of CNT growth: (i) catalyst deposition; (ii) thermal annealing and (iii) CNT growth.

from carbon contained in the atmosphere.

Depending on the relative surface energies of the support and the catalyst, re-structuration driven by de-wetting [18] of the catalyst film leads to CNT growth via the tip-growth mechanism (the catalyst particle still attached to the top of the nanotube) or via base-growth mechanism (the catalyst particle is attached to the substrate). As discussed above, annealing the catalyst at $973 \mathrm{~K}$ under $\mathrm{H}_{2}$ plasma produces a restructuration of the surface leading to self-organized catalyst particles [20]. This nanostructured surface is the key step of tip-growth CNTs synthesis because of its weak interaction with the substrate [15] [21] [22]. We conducted nanotube growth using a $10-\mathrm{cm}$-diameter vertical silica bell jar low-pressure reactor shown in Figure 2(a) with $\mathrm{CH}_{4}$ as the carbon precursor and $\mathrm{Si} / \mathrm{SiO}_{2}$ covered by the catalyst as substrate. For plasma generation, the reactor utilized a $1.2 \mathrm{~kW}$ microwave generator (SAIREM) operating at $2.45 \mathrm{GHz}$. The electromagnetic waves were generated, guided using a rectangular wave guide and directed into a cavity delimited by a Faraday cage. The short-circuit piston at the end of the wave guide helped create stationary waves and situate the maximum of the electric field near the substrate. Efficient operation was assumed with good microwave coupling and minimal radial diffusion to the quartz enclosure, leading to greater discharge stability and better plasma uniformity. Input power was varied with the pressure simultaneously in order to hold plasma volume constant.

As shown in Figure 2(b), a quasi-hemispherical active plasma zone of radius of $\sim 2.5 \mathrm{~cm}$ was created near the substrate. The input gases $\left(\mathrm{CH}_{4} / \mathrm{H}_{2} / \mathrm{O}_{2}\right)$ with electronically controlled mass flow rates were injected into the reactor and pumped out by the reactor pumping system. The substrate $\left(\sim 1 \times 1 \mathrm{~cm}^{2}\right.$ in size $)$ was placed on a resistive molybdenum boat (substrate holder). Substrate heating was achieved by flowing electric current from an external heating unit through a graphite resistor placed below the molybdenum holder. The substrate heating unit was designed to automatically adjust the current flow with respect to the temperature measured by the thermocouple embedded in the substrate holder. During all the experiments, the substrate temperature was monitored by an optical pyrometer (IMPAC, IGAQ-10), and we considered this temperature to be the actual substrate/deposition temperature for nanotube synthesis.

\section{Results and Discussion}

\subsection{Structural Analysis}

The influence of process parameter such as substrate temperature, microwave 


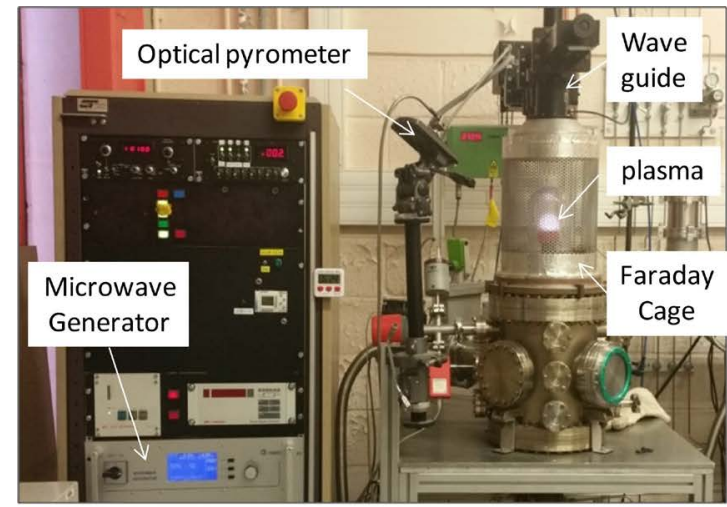

(a)

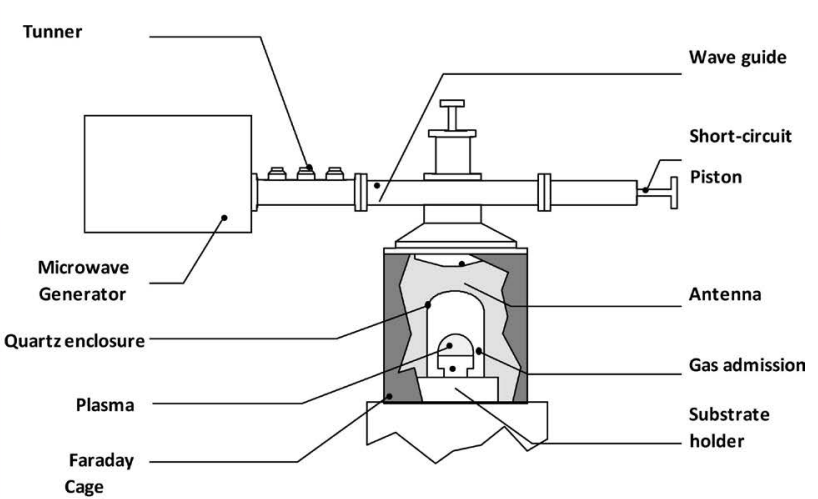

(b)

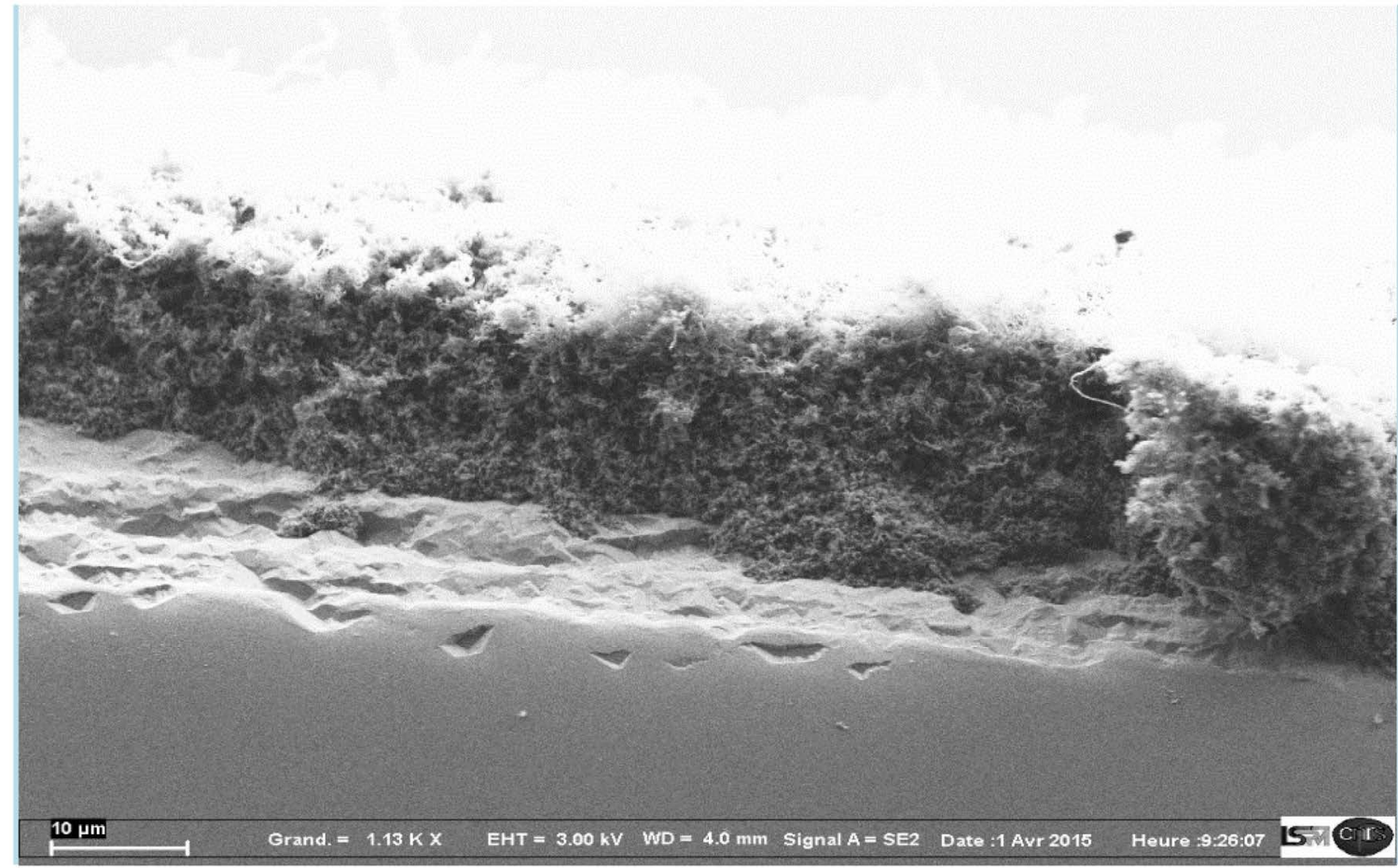

(c)

Figure 2. PECVD Bell jar reactor: (a) Photography of the experimental set-up used for nanotube growth; (b) Scheme of the CVD apparatus; (c) Typical SEM image of CNT forest obtained in this reactor. Conditions: pressure $=10 \mathrm{mbar}$; microwave power $=340$ watts; flow rates $\mathrm{H}_{2} / \mathrm{CH}_{4} / \mathrm{O}_{2}(90 \mathrm{sccm} / 10 \mathrm{sccm} / 2 \mathrm{sccm})$; substrate temperature $=923 \mathrm{~K}$.

plasma, gas composition and micro wave power on the structural quality and defect density in the graphitic lattice of the nanotube was ranked by monitoring the intensity of the D-bands relative to the G-band in the Raman spectra. After critical examination and adjusting of the conditions, optimum parameters have been determined for cobalt-palladium bimetals as: microwave power $=340$ watts; gas flow rates $\mathrm{H}_{2} / \mathrm{CH}_{4} / \mathrm{O}_{2}(10 \mathrm{sccm} / 10 \mathrm{sccm} / 2 \mathrm{sccm})$ and substrate temperature $=973 \mathrm{~K}$. A SEM image of as grown PECVD nanotube forest is shown in Figure 2(c) with forest height of $\sim 20 \mu \mathrm{m}$ for 20 min methane injection. Typical micro-Raman measurement of obtained CNTs forest is shown in Figure 3 with a G-band located at $\sim 1600 \mathrm{~cm}^{-1}$ and assigned to the in-plane vibration of the carbon-carbon bonds. In addition, a D-band located at $\sim 1340 \mathrm{~cm}^{-1}$ (D band) and 


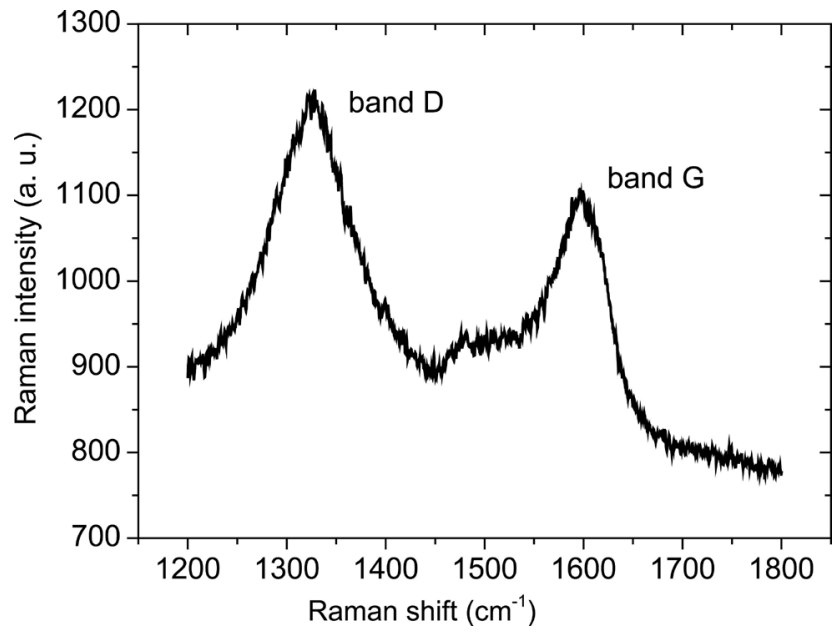

Figure 3. Typical Raman spectrum obtained. The G and D band are clearly visible and their ratio is 0.75 . Conditions: pressure $=10 \mathrm{mbar}$; microwave power $=340$ watts; flow rates $\mathrm{H}_{2} / \mathrm{CH}_{4} / \mathrm{O}_{2}(40 \mathrm{sccm} / 10 \mathrm{sccm} / 2 \mathrm{sccm})$; substrate temperature $=973 \mathrm{~K}$; substrate = $\mathrm{Si} / \mathrm{SiO}_{2} / \mathrm{Co}(3 \mathrm{~nm}) / \mathrm{Pd}(2 \mathrm{~nm})$.

is activated by the presence of disorder in carbon systems. The ratio between $\mathrm{D}$ peak and $G$ peak characterizes the structural quality of the CNTs.

In our case, it changes from 0.6 to 1 depending on growth conditions. Various experimental conditions were tried using palladium as co-catalyst and the best catalyst combination for the CNTs growth and filling was found Co $(3 \mathrm{~nm}) / \mathrm{Pd}$ $(2 \mathrm{~nm})$ with $\mathrm{H}_{2}$ plasma of $(10 \mathrm{sccm}), \mathrm{CH}_{4}(10 \mathrm{sccm}), \mathrm{O}_{2}(2 \mathrm{sccm})$ and an annealing temperature of $973 \mathrm{~K}$. At higher substrate temperatures, catalyst diffusion and nanostructuration are less efficient leading to a lack of CNTs growth. The presence of palladium as co catalyst with the cobalt enhances the nanotubes filling. This could be attributed to the presence of eutectic in $\mathrm{Co} / \mathrm{Pd}$ system that melt at lower temperature than pure Co or pure Pd and diffuses inside the MWCNT during the growth. A CNT filled with Co is shown in Figure 4 together with the element analysis of the metal inside the CNT. Because of the small size of the nanostructured catalyst, the CNT is filled on a few hundred nm. For the effective characterization of nanotubes, high resolution, spatially resolved Electron Energy Loss Spectroscopy (EELS), and energy filtered elemental mapping have proved the atomic distribution in the internal canal of the nanotube [23]. Figure 4(b) and Figure 4(c) show the energy-filtered TEM images of Co and Pd. It shows the presence of cobalt and palladium species which are separated into well-defined pure Co and Pd domains within the nanotube, independently of the catalyst stack [23]. The spatially resolved Co map is wider than that of Pd. It is also noted that Co map contrast is brighter in the interior of nanotubes; indeed Co is found mainly in the internal canal of the nanotubes.

The lack of orientation of the nanotubes is related to the disintegration of the catalyst films in nanoparticles. Under the effect of temperature, the atoms in the catalyst tends to diffuse into the volume generating instability in the nanotubes growth visible by the large number of defects and the low degree of alignment observed, in the absence of barrier layers. Thus, the "stabilization" of the catalyst 

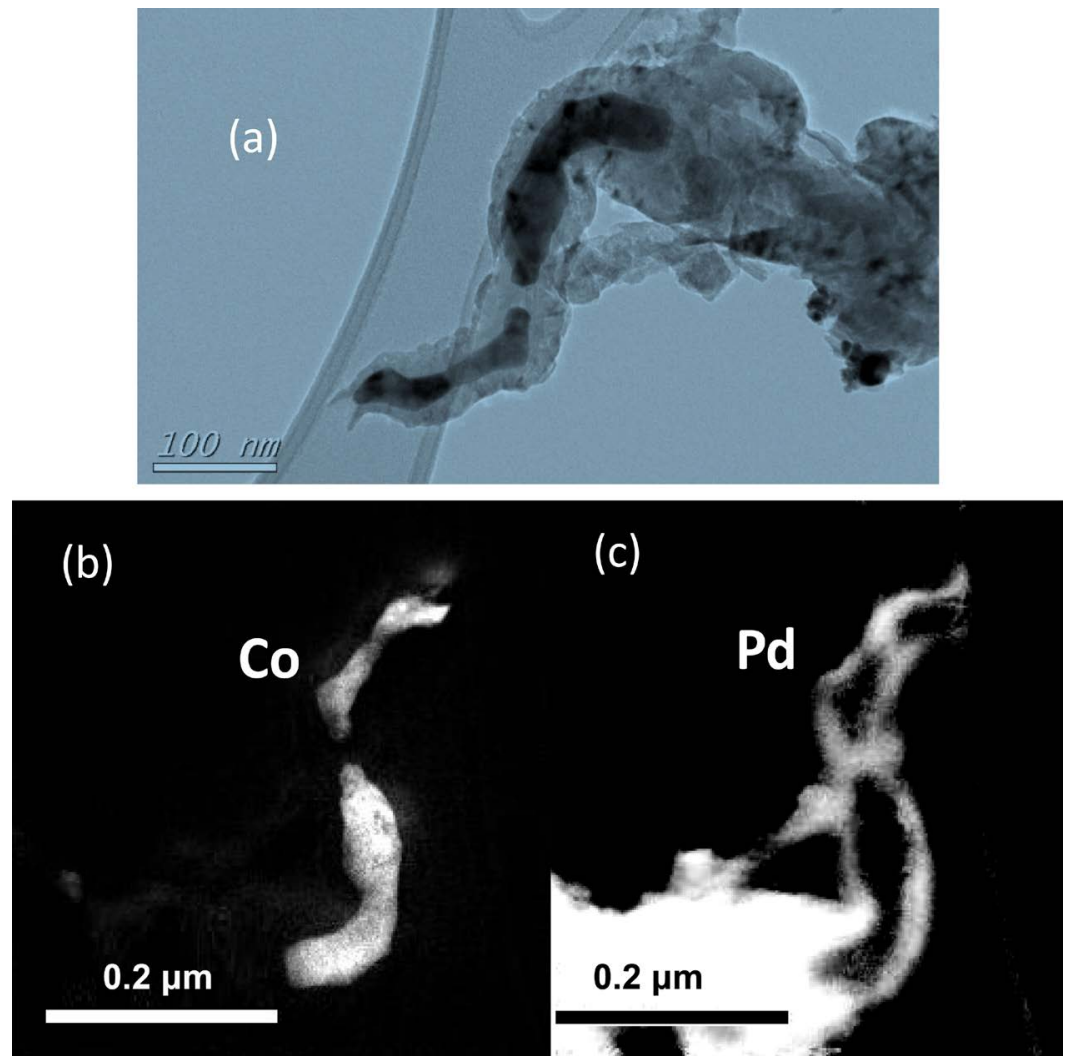

Figure 4. (a) Transmission electron microscope image of a MWCNT filled from a Co (3 $\mathrm{nm}) / \mathrm{Pd}(2 \mathrm{~nm})$ over a length of several hundred of $\mathrm{nm}$. Spatially resolved Electron Energy Loss Spectroscopy (EELS) of (b) cobalt and (c) palladium Conditions: substrate = $\mathrm{Si} / \mathrm{SiO}_{2} / \mathrm{Co}(3 \mathrm{~nm}) / \mathrm{Pd}(2 \mathrm{~nm})$; pressure $=10 \mathrm{mbar}$; microwave power $=340$ watts; flow rates $\mathrm{H}_{2} / \mathrm{CH}_{4} / \mathrm{O}_{2}(10 \mathrm{sccm} / 10 \mathrm{sccm} / 2 \mathrm{sccm})$; substrate temperature $=973 \mathrm{~K}$.

during the nanotubes growth appears to be a major technological barrier. In the work of Hata group in Japan, the alumina diffusion barrier $\left(\mathrm{Al}_{2} \mathrm{O}_{3}\right)$ allowed a breakthrough [17] [24]. More recently, for growing nanotubes on conductive substrates for applications such as heat sinks in microelectronic circuits, silicon-titanium nitride (TiSiN) have been proposed as a diffusion barrier by Robertson Group in Cambridge [25].

\subsection{Magnetic Measurements}

\subsubsection{MOKE Measurements}

Magnetic hysteresis of Co-Pd filled CNTs was determined from magnetization loop through magneto-optical Kerr measurements with a red laser. This technique describes the change of the polarization states of light when reflected at a magnetic material. Both polar (i.e. magnetization perpendicular to the plane) and planar magnetization of the filled MWCNTs are measured. In Figure 5, we represent the Kerr loop corresponding to the synthesized CNT forest. The polar hysteresis is almost rectangular; the remnant magnetization is thus perpendicular to the substrate in the main direction of the CNTs, whereas no planar magnetization is measured. One has to notice that even if $\mathrm{Pd}$ is present with $\mathrm{Co}$ as co-catalyst, magnetic response from Kerr measurements is obtained. This is 


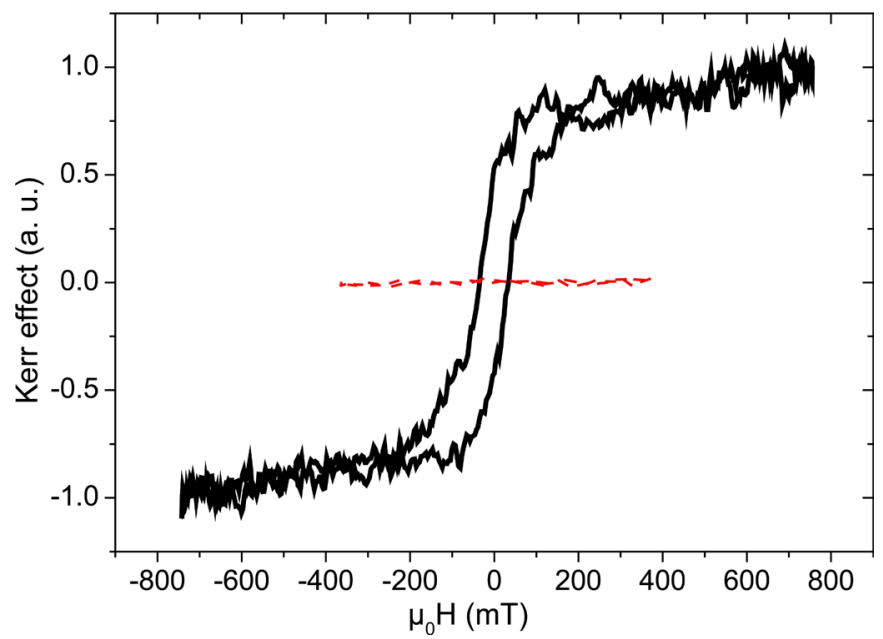

Figure 5. Polar (continuous curve) and planar (red dashed line) MOKE cycles obtained from CNT forest area of the sample.

expected since there is metal separation in the filled MWCNT as shown in Figure 4(b) and Figure 4(c). We also note that no direct correlation between MWCNT quality determined by the ratio $I_{G} / I_{D}$ and the magnetic behavior was observed. This was expected since the magnetic behavior of carbon and palladium is significantly weaker than cobalt. Another interesting feature from Figure 5 is the huge difference between longitudinal and polar measurements. This difference is discussed later with the help of superconducting quantum interference device (SQUID) measurements.

An interesting point to address is the difference in magnetic behavior that we have observed between the nanostructured catalyst obtained after annealing, without CNTs growth and the situation after growing filled CNTs. Figure 6 represented the polar and planar MOKE measurements of such nanostructured catalyst in the case of Co $(9 \mathrm{~nm}) / \mathrm{Pd}(6 \mathrm{~nm})$. One can observe that both magnetic loops have significant non zero hysteresis and a low remnant magnetization at zero field. This is similar to the hysteresis reported in [20], where changes in coercivity were found to depend on the size of the magnetic clusters, obtained from the nanostructuration process following the thermal annealing of the catalyst, as explained in Section 2. Moreover, as reported in [26], the possibility to form cobalt carbide would add a superpara magnetic behavior to the global magnetic answer. However, the resulting hysteresis is significantly less rectangular than that in presence of filled nanotubes.

\subsubsection{SQUID Measurements}

In order to get more insights on the magnetization behavior, we have used SQUID technique to provide magnetization measurement with good accuracy. It is then interesting to compare such magnetization measurements to those given by MOKE technique. We investigated the sample used for MOKE measurements. SQUID measurements were performed in plane and out of plane magnetization configurations at $10 \mathrm{~K}$ as shown in Figure 7. The hysteresis loops are similar for both magnetization directions. Moreover, they do not depend on temperature 


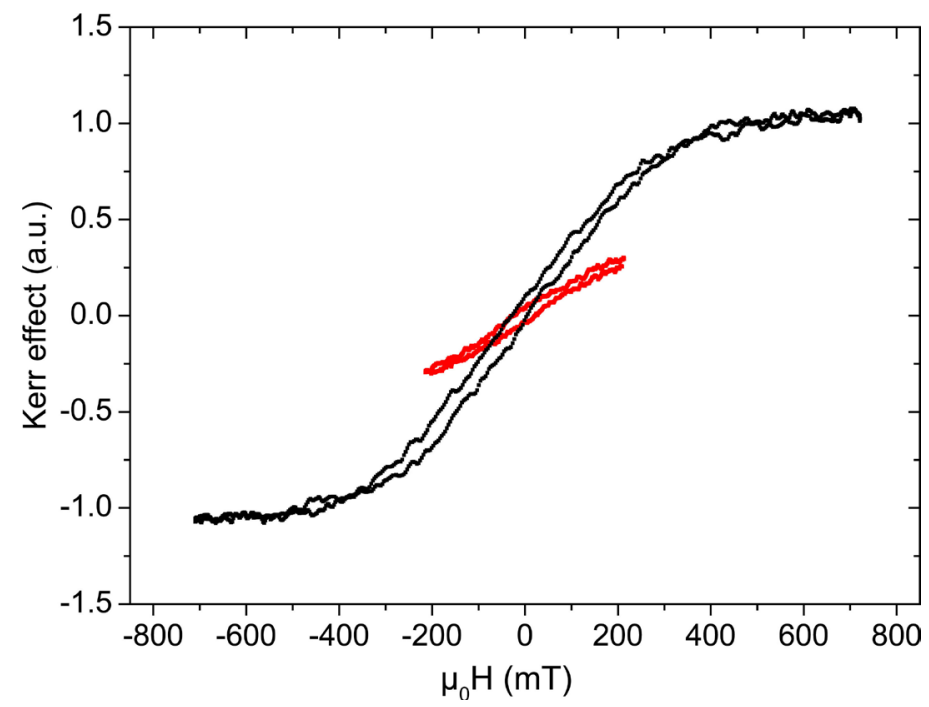

Figure 6. Polar (black) and planar (red) MOKE cycles of nanostructured surface obtained after unsuccessful CNT growth. Conditions: pressure $=10$ mbar; microwave power $=340$ watts; flow rates $\mathrm{H}_{2} / \mathrm{CH}_{4} / \mathrm{O}_{2}(10 \mathrm{sccm} / 10 \mathrm{sccm} / 2 \mathrm{sccm})$; substrate temperature $=1023 \mathrm{~K}$.

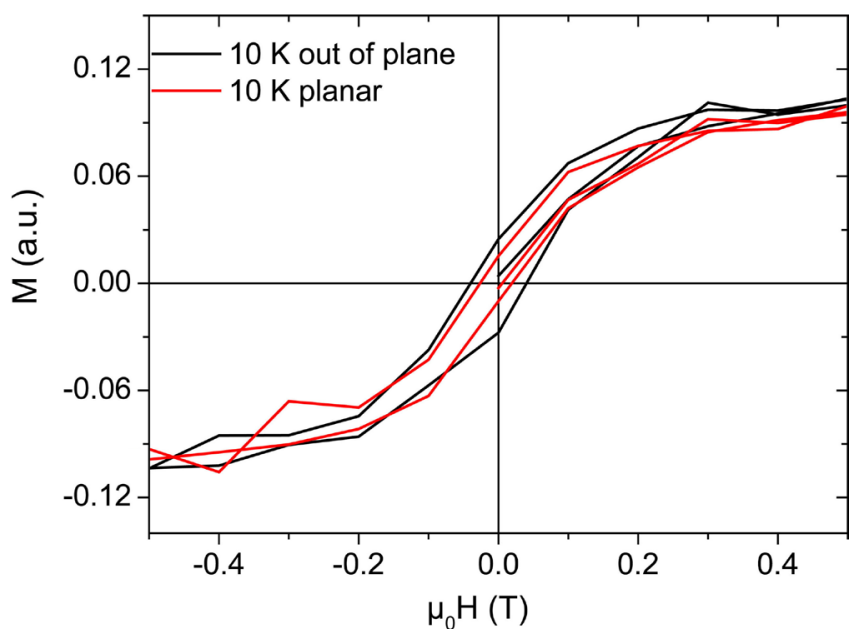

Figure 7. Planar (red) and out of plane i.e. along the main CNTs axis (black) SQUID measurements of the filled CNTs. The out of plane measurement has been dilated to match its amplitude with the planar measurement.

between $10 \mathrm{~K}$ and $290 \mathrm{~K}$. The behavior is a mainly superparamagnetic-like with a small remnant magnetization at zero fields. To explain this difference with the MOKE measurements presented earlier, where a clear ferromagnetic behavior was observed, it is important to consider each method characteristics. The main difference between MOKE and SQUID measurements is how much volume of the sample can be measured. SQUID is global measurement of the whole sample while MOKE is more local technique. In case of MOKE, a laser with a finite spot size is used and can only sense a part of the thickness of the nanotubes. As shown in the TEM image in Figure 4, the CNT is filled over hundred nanometers but is not straight on the whole length. The MOKE measurement probes only the first $100 \mathrm{~nm}$ with a given direction of the nanotube while the SQUID measures the whole magnetic behavior with all possible orientations. Such dis- 
persion in CNT direction is also in good agreement with the SEM cross section observed in Figure 2(c) and in previously reported experiment with catalytic CVD [27]. Thus, all CNTs can be individually ferromagnetic but collectively the dispersion of their orientations gives rise to a superparamagnetic-like behavior in the global SQUID measurement. Conversely, the local MOKE technique probes an area where CNTs are more homogeneous and perpendicular to the surface of the substrate, especially in the finite thickness about $100 \mathrm{~nm}$ that is really probed.

Thus, SQUID and MOKE measurements give complementary information on the magnetism of filled CNTs at two different scales. One has to take into consideration this scale aspect for an unbiased analysis of the magnetic measurements. A last point to be noted from SQUID measurement reported in Figure 7 is that the hysteresis loop in out of plane configuration is slightly more hysteretic than the planar one. This indicates that, despite the high dispersion of the nanotubes orientations, the SQUID gives an indication of a direction of the anisotropy.

\section{Conclusion}

We successfully synthesized CNT forest with PECVD using intermetallic Co/Pd catalysts. Regarding structural nanotubes properties obtained from Raman spectra, the best CNT properties are achieved with Co $(3 \mathrm{~nm}) / \mathrm{Pd}(2 \mathrm{~nm})$ and a temperature of $973 \mathrm{~K}$. During the synthesis, the catalyst melts because of its eutectic composition and thus diffuses inside the MWCNTs over a few hundred nanometers, filling them uniformly with the ferromagnetic material. Locally from MOKE measurement, ferromagnetic loop is measured. However, global SQUID measurement gives superparamagnetic-like behavior, it averages the magnetic signal on the whole MWCNT forest with all the different CNT orientations. This scale aspect should be taken into account for an unbiased analysis of the magnetic measurements. Improvement of the MWCNT orientation will improve the magnetic properties. The filling of MWCNTs during PECVD synthesis is promising to provide partially functionalized magnetic MWCNTs for spintronic application where the ferromagnetic part of the CNT would be similar to a ferromagnetic electrode.

\section{Acknowledgements}

We thank S. Suffit for the catalyst deposition, A. Mougin for help in MOKE measurements and M. Viret for the SQUID measurements. IFR Paris Nord Plaine de France (PPF), ANR (Agence Nationale de la Recherche) and CGI (Commissariat à l'Investissement d'Avenir) are gratefully acknowledged for their financial support of this work through Labex SEAM (Science and Engineering for Advanced Materials and devices) ANR 11 LABEX 086, ANR 11 IDEX 05 02, HEFOR project (PRS-G-2014).

\section{References}

[1] Ajayan, P.M. and Iijima, S. (1993) Capillarity-Induced Filling of Carbon Nanotubes. Nature, 361, 333-334. https://doi.org/10.1038/361333a0 
[2] Tsang, S.C., Harris, P.J.F. and Green, M.L.H. (1993) Thinning and Opening of Carbon Nanotubes by Oxidation Using Carbon Dioxide. Nature, 362, 520-522. https://doi.org/10.1038/362520a0

[3] Gao, Y., Liu, J., Shi, M., Elder, S.H. and Virden, J.W. (1999) Dense Arrays of Well-Aligned Carbon Nanotubes Completely Filled with Single Crystalline Titanium Carbide Wires on Titanium Substrates. Applied Physics Letters, 74, 3642 3644. https://doi.org/10.1063/1.123208

[4] Kikkawa, J.M. and Awschalom, D.D. (1999) Lateral Drag of Spin Coherence in Gallium Arsenide. Nature, 397, 139-141. https://doi.org/10.1038/16420

[5] Tsukagoshi, K., Alphenaar, B.W. and Ago, H. (1999) Coherent Transport of Electron Spin in a Ferromagnetically Contacted Carbon Nanotube. Nature, 401, 572 574. https://doi.org/10.1038/44108

[6] Iijima, S. (1991) Helical Microtubules of Graphitic Carbon. Nature, 354, 56-58. https://doi.org/10.1038/354056a

[7] Journet, C., Maser, W.K., Bernier, P., Loiseau, A., de la Chapelle, M.L., Lefrant, S., Deniard, P., Lee, R. and Fischer, J.E. (1997) Large-Scale Production of SingleWalled Carbon Nanotubes by the Electric-Arc Technique. Nature, 388, 756-758. https://doi.org/10.1038/41972

[8] Guo, T., Nikolaev, P., Thess, A., Colbert, D.T. and Smalley, R.E. (1995) Catalytic Growth of Single-Walled Manotubes by Laser Vaporization. Chemical Physics Letters, 243, 49-54. https://doi.org/10.1016/0009-2614(95)00825-O

[9] Li, W.Z., Xie, S.S., Qian, L.X., Chang, B.H., Zou, B.S., Zhou, W.Y., Zhao, R.A. and Wang, G. (1996) Large-Scale Synthesis of Aligned Carbon Nanotubes. Science, 274, 1701-1703. https://doi.org/10.1126/science.274.5293.1701

[10] Li, J., Papadopoulos, C. and Xu, J.M. (1999) Highly-Ordered Carbon Nanotube Arrays for Electronics Applications. Applied Physics Letters, 75, 367-369.

https://doi.org/10.1063/1.124377

[11] Esconjauregui, S., Bhardwaj, S., Yang, J., Castellarin-Cudia, C., Xie, R., D’Arsié, L., Makaryan, T., Sugime, H., Eslava, S., Cepek, C. and Robertson, J. (2014) Carbon Nanotube Growth on Conductors: Influence of the Support Structure and Catalyst Thickness. Carbon, 73, 13-24. https://doi.org/10.1016/j.carbon.2014.02.026

[12] Stojak Repa, K., Israel, D., Alonso, J., Phan, M.H., Palmero, E.M., Vasquez, M. and Srikanth, H. (2015) Superparamagnetic Properties of Carbon Nanotubes Filled with $\mathrm{NiFe}_{2} \mathrm{O}_{4}$ Nanoparticles. Journal of Applied Physics, 117, $17 \mathrm{C} 723$. https://doi.org/10.1063/1.4914952

[13] Hayashi, Y., Tokunaga, T., Iijima, T., Iwata, T., Kalita, G., Tanemura, M., Sasaki, K. and Kuroda, K. (2012) High Temperature In-Situ Observations of Multi-Segmented Metal Nanowires Encapsulated within Carbon Nanotubes by In-Situ Filling Technique. Nanoscale Research Letters, 7, 448.

https://doi.org/10.1186/1556-276X-7-448

[14] Leonhardt, A., Hampel, S., Müller, C., Mönch, I., Koseva, R., Ritschel, M., Elefant, D., Biedermann, K. and Büchner, B. (2006) Synthesis, Properties, and Applications of Ferromagnetic-Filled Carbon Nanotubes. Chemical Vapor Deposition, 12, 380 387. https://doi.org/10.1002/cvde.200506441

[15] Ren, Z.F., Huang, Z.P., Xu, J.W., Wang, J.H., Bush, P., Siegal, M.P. and Provencio, P.N. (1998) Synthesis of Large Arrays of Well-Aligned Carbon Nanotubes on Glass. Science, 282, 1105-1107. https://doi.org/10.1126/science.282.5391.1105

[16] Meshot, E.R., Plata, D.L., Tawfick, S., Zhang, Y.Y., Verploegen, E.A. and Hart, A.J. (2009) Engineering Vertically Aligned Carbon Nanotube Growth by Decoupled 
Thermal Treatment of Precursor and Catalyst. ACS Nano, 3, 2477-2486. https://doi.org/10.1021/nn900446a

[17] Hata, K., Futaba, D.N., Mizuno, K., Namai, T., Yumura, M. and Iijima, S. (2004) Water-Assisted Highly Efficient Synthesis of Impurity-Free Single-Walled Carbon Nanotubes. Science, 306, 1362-1364. https://doi.org/10.1126/science.1104962

[18] Rupprechter, G. (2016) Chap. 39: Surface Science Approach to Heterogeneous Catalysis. In: Wandelt, K., Ed., Surface and Interface Science, Volume 5: Solid-Gas Interfaces I, Wiley-VCH Verlag GmbH \& Co. KGaA, Weinheim, Germany. https://doi.org/10.1002/9783527680573.ch39

[19] Borowiak-Palen, E., Mendoza, E., Bachmatiuk, A., Rummeli, M.H., Gemming, T., Nogues, J., Skumryev, V., Kalenczuk, R.J., Pichler, T. and Silva, S.R.P. (2006) Iron Filled Single-Wall Carbon Nanotubes-A Novel Ferromagnetic Medium. Chemical Physics Letters, 421, 129-133. https://doi.org/10.1016/j.cplett.2006.01.072

[20] Bounour-Bouzamouche, W., Chérif, S.M., Farhat, S., Roussigné, Y., Tallaire, A., Gicquel, A., Lungu, C.P. and Guerioune, M. (2014) Structural and Magnetic Properties of Cobalt Nanostructures on $\mathrm{SiO}_{2} / \mathrm{Si}\left(\begin{array}{lll}1 & 1 & 1\end{array}\right)$ Substrates. Applied Surface Science, 320, 858-862. https://doi.org/10.1016/j.apsusc.2014.09.148

[21] Park, J.-B., Cho, Y.-S., Hong, S.Y., Choi, K.S., Kim, D., Choi, S.-Y., Ahn, S.-D., Song, Y.-H., Lee, J.-H. and Cho, K.-I. (2002) Cross-Sectional Transmission Electron Microscopy of Carbon Nanotubes-Catalyst-Substrate Heterostructure Using a Novel Method for Specimen Preparation. Thin Solid Films, 415, 78-82. https://doi.org/10.1016/S0040-6090(02)00507-2

[22] Choi, H.C., Kundria, S., Wang, D., Javey, A., Wang, Q., Rolandi, M. and Dai, H. (2003) Efficient Formation of Iron Nanoparticle Catalysts on Silicon Oxide by Hydroxylamine for Carbon Nanotube Synthesis and Electronics. Nano Letters, 3, 157 161. https://doi.org/10.1021/nl025876d

[23] Hayashi, Y., Fujita, T., Tokunaga, T., Kaneko, K., Butler, T., Rupesinghe, N., Carey, J.D., Silva, S.R.P. and Amaratunga, G.A.J. (2007) Encapsluation of Co and Pd Multi-Metal Nanowires Inside Multiwalled Carbon Nanotubes by Microwave Plasma Chemical Vapor Deposition. Diamond and Related Materials, 16, 1200-1203. https://doi.org/10.1016/j.diamond.2006.11.039

[24] Hata, K. (2015) A Sweet Spot for Highly Efficient Growth of Vertically Aligned SWNT Forests. Guadalupe Workshop VII on Single Wall Carbon Nanotube Nucleation and Growth Mechanisms, San Antonio, 10-14 April 2015.

[25] Yang, J., Esconjauregui, S., Robertson, A.W., Guo, Y., Hallam, T., Sugime, H., Zhong, G., Duesberg, G.S. and Robertson, J. (2015) Growth of High-Density Carbon Nanotube Forests on Conductive TiSiN Supports. Applied Physics Letters, 106, Article ID: 083108. https://doi.org/10.1063/1.4913762

[26] Huh, S.H. and Nakajima, A. (2006) Laser Synthesis and Magnetism of Amorphous Iron and Cobalt Carbide Nanoparticles with Carbon Onion. Journal of Applied Physics, 99, Article ID: 064302. https://doi.org/10.1063/1.2174116

[27] Leonhardt, A., Ritschel, M., Kozhuharova, R., Graff, A., Mühl, T., Hunhle, R., Mönch, L., Elefant, D. and Schneider, C.M. (2003) Synthesis and Properties of Filled Carbon Nanotubes. Diamond and Related Materials, 12, 790-793. https://doi.org/10.1016/S0925-9635(02)00325-4 